\title{
Correlação das escalas de avaliação utilizadas na doença de Parkinson com aplicabilidade na fisioterapia
}

\author{
Correlation of evaluation scales utilitized at Parkinson's \\ disease applied to physical therapy
}

\author{
Marcella Patrícia Bezerra de Mello ${ }^{[a]}$, Ana Carla Gomes Botelho ${ }^{[\mathrm{b}]}$
}

[a] Especialista em Fisioterapia Neurofuncional pela Faculdade Integrada do Recife (FIR), Departamento de Fisioterapia Neurofuncional, Especialista em Saúde Pública com Ênfase em Gerência de Serviços e Saúde da Família pela Faculdade de Enfermagem Nossa Senhora das Graças (FENSG/UPE), Recife, PE - Brasil, e-mail: marcellapb@hotmail.com

b] Especialista em Fisioterapia em Neonatologia e Pediatria pela Faculdade Integrada do Recife (FIR), Departamento de Fisioterapia Neurofuncional, Mestranda em Saúde Materno-Infantil pelo Instituto de Medicina Integral Prof. Fernando Figueira (IMIP), Recife, PE - Brasil, e-mail: anacbotelho@hotmail.com

\section{Resumo}

Introdução: A doença de Parkinson (DP) é uma patologia neurológica crônica e degenerativa do sistema nervoso central que acomete os gânglios da base, cujas características principais são tremor, rigidez e bradicinesia. Com o progresso terapêutico, desenvolveram-se várias escalas visando monitorar a evolução da doença e a eficácia de tratamentos. O objetivo deste estudo de revisão bibliográfica é caracterizar as principais escalas usadas para a avaliação da DP, discutindo sua aplicabilidade à prática fisioterapêutica. Materiais e método: Levantamento bibliográfico a partir de bases de dados como Scielo, Medline, Lilacs, PubMed, entre 1990 a 2005. Resultados: Seis escalas são abordadas: Escala dos Estágios de Incapacidade de Hoehn e Yahr; Escala Unificada de Avaliação da DP (UPDRS); Escala de Sydney; Questionário da DP (PDQ-39); Qualidade de vida (PSN); Escala de atividade de Parkinson (PAS). Destacando-se a PDQ-39 pela percepção do paciente sobre sua qualidade de vida. A PAS é a que melhor atende aos objetivos específicos da fisioterapia, pois avalia os principais problemas de mobilidade funcional. Além das escalas de Hoehn e Yahr e a UPDRS, por sua confiabilidade, pois podem ser usadas por fisioterapeutas para melhor avaliação do estado clínico-funcional do paciente. Conclusão: A necessidade de monitorar a evolução dos pacientes e os resultados de intervenção fisioterapêutica exige do fisioterapeuta o conhecimento para utilizar medidas sistematizadas e de fácil aplicabilidade para avaliar pacientes com DP. Cabe a esse profissional optar pela que permita uma tomada de decisão clínica compatível com seu local de trabalho, com as necessidades do paciente e com o meio em que ele vive.

Palavras-chave: Doença de Parkinson. Escalas de Avaliação. Fisioterapia. Avaliação. Qualidade de vida. 


\begin{abstract}
Introduction: The Parkinson's disease (PD) is a degenerative illness of the central nervous system, the key features are tremor, rigidity and bradykinesia. Therapeutic progress has brought about several clinical scales to assess PD patients, in an attempt to monitor disease development and to evaluale the affectiveness about different treatments. The objective of this bibliographic revision study is to characterize the main scales used to valuation of the $P D$, discussing their application onto physical therapy practice. Materials and method: A bibliographical survey through of books, scientific articles and database specialized to acquisition of newspapers and sites of Internet. Results: Six scales are focused: The Scale of Hoehn and Yahr about Incapacity Stages; Unified Parkinson's Disease Rating Scale (UPDRS); Sydney Scale; PD Questionnaire (PDQ-39) and Quality of Life (PSN); and Parkinson's Activity Scale (PAS).The PDQ-39 stand out because of the patient perception about their quality of life. The PAS is the best attend the physical therapy objectives, because of evaluate the main problems of functional mobility. In addition to Hoehn and Yahr scale and the UPDR scale because of their reliability and may be used by physical therapists, allowing the best evaluation of patient's functional status. Conclusion: The follow the evolution of patients and their results about physical therapies interventions require knowledge of the physical therapists to make systematized decisions, and easy applications to value patients DP. The professional must make a clinical decision compatible at his job place, patient necessity and his environment that he lives.
\end{abstract}

Keywords: Parkinson's disease. Rating Scale. Physical Therapy. Evaluation. Quality of life.

\title{
Introdução
}

A doença de Parkinson (DP) é uma patologia neurológica crônica e degenerativa do sistema nervoso central que acomete os gânglios da base (1). A DP é caracterizada pela redução de dopamina na via negro-estriatal, resultante da morte de neurônios da substância negra cerebral. A etiologia específica não é conhecida, porém, durante os últimos anos, tem-se considerado fatores hereditários, infecciosos, tóxicos, genéticos e ambientais (2).

É uma doença lentamente progressiva e comum em pessoas idosas, pois afeta 1 em cada 1.000 indivíduos acima de 65 anos e 1 em cada 100 após os 75 anos (2, 3), segundo a Organização Mundial de Saúde (OMS). Homens são mais afetados que as mulheres na proporção de 2:1 (4).

Os principais sinais e sintomas da DP caracterizam-se por lentidão nas tarefas motoras, tremor ao repouso, rigidez, bradicinesia e alterações da postura, do equilíbrio e da marcha. Além disso, os pacientes com DP podem apresentar alterações músculo-esqueléticas, como fraqueza e encurtamento muscular, alterações neurocomportamentais, como demência, depressão e tendência ao isolamento e comprometimento cardiorrespiratório, o que interfere diretamente na performance funcional e independência desses indivíduos (5).

Com o desenvolvimento de novos tratamentos para a DP, tornou-se necessário criar e desenvolver escalas para avaliar a doença (6). Essas escalas avaliam desde a condição clínica geral, incapacidades, função motora e mental até a qualidade de vida dos pacientes. Tais instrumentos são importantes tanto clínica quanto cientificamente, pois permitem monitorar a progressão da doença e a eficácia de tratamentos e drogas (7).

A fisioterapia adquire um importante papel na reabilitação desses pacientes, cujos objetivos passam por minimizar e retardar a evolução dos sintomas, melhorar a mobilidade, a força muscular, o equilíbrio, a aptidão física, proporcionando uma evolução da funcionalidade e consequente melhoria da qualidade de vida $(8,9)$. Contudo, segundo duas revisões sistemáticas realizadas por Deane et al. (10), não há evidência suficiente para suportar ou refutar a eficácia da fisioterapia e das suas técnicas na DP. 
Para que se consiga atingir esses objetivos, faz-se necessário a realização de uma criteriosa avaliação do paciente, com o intuito de determinar o seu real nível de comprometimento, uma vez que, por a DP ser uma patologia degenerativa e progressiva, a intervenção tem de ser adequada às reais necessidades (11-13).

Existem várias escalas de avaliação que contribuem para a melhor elaboração de um plano de reabilitação, bem como acompanhamento dos pacientes no curso de desenvolvimento da doença, visando monitorar a sua evolução (14).

Para a medição ser capaz de avaliar a eficácia de um tratamento e ter credibilidade científica, deve apresentar determinadas propriedades psicométricas como confiabilidade, validade e sensibilidade. Uma medição é considerada confiável quando produz resultados precisos, consistentes e reproduzíveis. Quanto maior a confiabilidade de uma medida, maior a segurança que se tem ao fazer julgamentos com base nela (15).

A validade é definida como a evidência de que um teste mede o que se propõe a medir, bem como se a medida obtida pode ser legitimamente usada para se fazer inferências (14). No processo de validação de uma medida ou instrumento, é importante observar que a validade deve ser formalmente comprovada por uma evidência concreta, essa evidência deve ser obtida usando métodos específicos (16). Já a sensibilidade é a característica de uma medida ou instrumento de detectar mudanças clinicamente significativas (15).

Assim, o objetivo deste estudo é descrever as principais escalas usadas para a avaliação da DP, discutindo sua aplicabilidade à prática fisioterapêutica, visto que os fisioterapeutas raramente utilizam instrumentos para avaliar especificamente a DP, uma vez que os instrumentos disponíveis são pouco difundidos em seu meio. No entanto, esses fatores, associados à necessidade de monitorar a evolução dos pacientes e buscar evidências científicas para embasar os diferentes tipos de intervenções terapêuticas, demonstram a relevância de se conhecerem as escalas de avaliação para a DP e suas possíveis aplicações na fisioterapia.

\section{Materiais e método}

O presente estudo trata-se de uma revisão normativa, realizada a partir do levantamento bibliográfico através de artigos indexados nas bases de dados SciELO, Medline, Lilacs, pelos quais se estabeleceram como critérios de inclusão estudos realizados entre 1990 a 2005, nos idiomas inglês e português, e revisões sistemáticas. Além disso, foram incluídas no estudo duas referências com relevância sobre o tema, datadas de 1985. Tendo como objetivo recolher informações sobre instrumentos avaliativos utilizados na DP, bem como suas evidências no que diz respeito à credibilidade científica e aplicabilidade fisioterapêutica.

Foram encontrados 21 artigos que corresponderam aos critérios de inclusão, cujos títulos continham os seguintes descritores: doença de Parkinson (Parkinson's disease), escala de avaliação (rating scale), fisioterapia (physical therapy), qualidade de vida (quality of life).

\section{Resultados}

\section{Principais escalas para avaliação da doença de Parkinson}

Escala de Hoehn e Yahr Modificada

Escala de Estágios de Incapacidade de Hoehn e Yahr (HY - Degree of Disability Scale) é uma escala de avaliação da incapacidade dos indivíduos com DP capaz de indicar seu estado geral de forma rápida e prática. Sua forma modificada compreende sete estágios de classificação para avaliar a gravidade da DP e abrange, essencialmente, medidas globais de sinais e sintomas que permitem classificar o indivíduo quanto ao nível de incapacidade. Os indivíduos classificados nos estágios de 1 a 3 apresentam incapacidade leve a moderada, enquanto os que estão nos estágios 4 e 5 apresentam incapacidade grave (17). 


\section{Escala Sydney}

Foi usada pela primeira vez em 1984, para portadores da DP, se subdivide em 11 categorias, com um escore total de 89. As categorias compreendem itens como expressão facial, seborreia, sialorreia, fala, levantar-se de uma cadeira, postura, estabilidade postural, marcha, tremor e tremor postural, destreza digital e rigidez. Quanto menor a pontuação, melhor a condição do paciente (6).

Escala unificada de avaliação da doença de Parkinson (UPDRS)

Essa escala avalia os sinais, sintomas e determinadas atividades dos pacientes por meio do autorrelato e da observação clínica. É composta por 42 itens, divididos em quatro partes: atividade mental, comportamento e humor; atividades de vida diária (AVD's); exploração motora e complicações da terapia medicamentosa. A pontuação em cada item varia de 0 a 4 , sendo que o valor máximo indica maior comprometimento pela doença e o valor mínimo indica tendência à normalidade $(18,19)$.

Questionário de doença de Parkinson (PDQ-39)

O PDQ-39 é uma escala específica para a DP e compreende 39 itens que podem ser respondidos com cinco opções diferentes de resposta: "nunca, de vez em quando, às vezes, frequentemente e sempre ou impossível para mim". Ele é dividido em oito categorias: mobilidade (10 itens), atividades de vida diária (6 itens), bem-estar emocional (6 itens), estigma (4 itens), apoio social (3 itens), cognição (4 itens), comunicação (3 itens) e desconforto corporal (3 itens). A pontuação varia de 0 (nenhum problema) a 100 (máximo nível de problema), ou seja, baixa pontuação indica a percepção do indivíduo de uma melhor qualidade de vida $(20,21)$.

Escala de atividade de Parkinson (PAS)

É uma escala para caracterizar problemas funcionais de indivíduos que estão nos estágios moderado e severo da doença. O conteúdo da PAS reflete alguns problemas de movimento na DP, tais como dificuldade de controlar o centro de massa corporal quando levantando de uma cadeira, hesitação, festinação ou freezing na marcha, limitação da mobilidade axial e dificuldade em realizar movimentos complexos, como fazer duas tarefas ao mesmo tempo. Os itens da escala são divididos em quatro categorias: transferências na cadeira, acinesia na marcha, mobilidade na cama e mobilidade na cama com uso do cobertor. O escore varia de zero a quatro em cada categoria, de modo que uma pontuação máxima indica melhor condição do paciente e a mínima indica que o indivíduo necessita de ajuda física (22).

Questionário de qualidade de vida da doença de Parkinson (PSN)

A qualidade de vida foi medida pelo perfil de Saúde de Notthingham (PSN), um indicador simples e direto da percepção do indivíduo em relação à saúde física, emocional e social. Este instrumento de avaliação é um questionário com 38 questões e duas alternativas de respostas (sim ou não). Envolve questões como habilidade física, nível de energia, dor, reações emocionais, isolamento social e qualidade do sono. Cada resposta "sim" corresponde a um ponto e quanto menor a pontuação total obtida, melhor a percepção do indivíduo em relação à sua qualidade de vida (23).

\section{Discussão}

\section{Uso das escalas avaliativas da DP e sua aplicabilidade na prática fisioterapêutica}

No contexto da doença crônica, o fisioterapeuta busca diminuir a disfunção física e permitir ao indivíduo realizar atividades de seu dia-a-dia com maior eficiência e independência possível (24). Com isso, a avaliação dos pacientes com DP requer instrumentos e medidas apropriadas que abordem tanto aspectos mais genéricos, como força muscular e amplitude de movimento. É importante que as avaliações possam monitorar as mudanças funcionais em todos os estágios da doença e, ao mesmo tempo, sejam sensíveis principalmente no que diz respeito à intervenção terapêutica (25). 
Num estudo realizado com 20 indivíduos sadios e 20 indivíduos portadores de DP, utilizando uma comparação de variáveis funcionais e de qualidade de vida, em que as variáveis foram: velocidade da marcha, descer e subir escadas e a escala de qualidade de vida PSN, os resultados demonstraram redução significativa da velocidade da marcha, como também foram mais lentos para subir e descer escadas quando comparados ao grupo controle. Tais resultados sugerem menor independência desses indivíduos, mesmo em estágios inicial e moderado da doença pela depleção de dopamina nos gânglios basais. Indivíduos com DP experimentam redução progressiva na velocidade e amplitude de movimento (4), sendo a bradicinesia a principal desordem motora percebida na doença (2). Já com relação à qualidade de vida, os indivíduos com DP apresentam escore do PSN mais alto do que os adultos e idosos, sugerindo pior percepção dos pacientes com relação a sua própria qualidade de vida.

Em seus estudos, Nieuwboer et al. (22) afirmam que a escala PAS parece ser a única desenvolvida, até o momento, para atender objetivos específicos da fisioterapia para avaliar os principais problemas de mobilidade funcional que acometem o indivíduo nos estágios moderado a avançado da DP. Pelo fato de o conteúdo dessa escala refletir alguns problemas de movimento na doença, tais como dificuldades de controlar o centro de massa corporal, festinação, dificuldade em realizar movimentos complexos e mobilidade axial.

Como escalas de avaliação fisioterapêutica específicas para a DP não estão disponíveis, vale ressaltar a HY e a UPDRS por suas características. A primeira permite ao terapeuta conhecer o estágio da doença em que o paciente se encontra e a obtenção de um breve resumo de seus sinais e sintomas (26), classificando o estado geral do indivíduo, porém é uma escala superficial, com pouca sensibilidade para detectar mudanças clínicas nos pacientes. Apesar da praticidade e ampla utilização, apresenta baixa sensibilidade à mudança e baixa confiabilidade, principalmente para os estágios precoces da doença (18).

Já a UPDRS também é muito utilizada e, apesar da alta confiabilidade, tem a desvantagem de ser extensa, exigindo um tempo maior para sua aplicação, entretanto, permite documentar também algumas habilidades funcionais nos itens de exploração motora e AVD's, monitorando o paciente ao longo do curso da doença (18). Os resultados de um estudo realizado por Van Hilten et al. (7) mostram que as variáveis sobre AVD's e exploração motora podem ser reduzidas para oito itens cada, sem diminuir a confiabilidade e validade da UPDRS.

Essas escalas, apesar de não terem sido desenvolvidas para aplicação em fisioterapia são mundialmente conhecidas, confiáveis, validadas e podem ser úteis ao fisioterapeuta, haja vista a escassez de instrumentos específicos na área. Por outro lado, a escala de Sydney apresenta limitações quanto a suas características psicométricas, por causa dessa escala ter sensibilidade desconhecida (6).

Uma possível limitação da análise da qualidade de vida feita em estudos refere-se ao fato do PSN ser um questionário genérico, ou seja, a utilização de instrumentos específicos para a DP deve permitir avaliar melhor o verdadeiro impacto dessa doença na qualidade de vida dos indivíduos $(27,28)$.

Atualmente, tem-se dado ênfase às escalas de qualidade de vida que apresentam a vantagem de avaliar mais amplamente o bem-estar geral dos pacientes. O PDQ-39 foi desenvolvido com o intuito de produzir um instrumento curto, simples e prático para avaliar a qualidade de vida dos pacientes com DP (29). Contudo, a análise estatística e interpretação dos dados foram apontadas como um problema, por sua complexidade potencial (30). Entretanto, esse instrumento não pode ser aplicado a pacientes com déficits cognitivos significativos (31).

\section{Considerações finais}

A necessidade de monitorar a evolução dos pacientes e os resultados de intervenção fisioterapêutica exige que o fisioterapeuta conheça e utilize medidas sistematizadas e de fácil aplicabilidade para avaliar pacientes com DP. Como visto, várias escalas podem ser usadas para esse fim e cabe ao profissional optar por aquela ou aquelas que permitam uma tomada de decisão clínica compatível com seu local de trabalho, com as necessidades do paciente e com o meio em que ele vive. 


\section{Referências}

1. $\quad$ Agid Y. Parkinson's disease pathophysiology. Lancet. 1991;337(8753):1321-4.

2. Morris ME. Movement disorders in people with parkinson disease: a model for physical therapy. Phys Ther. 2000;80(6):578-97.

3. Cutson TM, Laub KC, Schenkman M. Pharmacological and nonpharmacological interventions in the treatment of Parkinson's disease. Phys Ther. 1995;75(5):363-73.

4. Marsden CD. Parkinson‘s disease. J Neurol Neurosurg Psychiatry. 1994;57(6):672-81.

5. Canning CG, Alison JA, Allen NE, Groller H. Parkinson's disease: an investigation of exercise capacity, respiration function, and gait. Arch Phys Med Rehabil. 1997;78(2):199-207.

6. Hely MA, Chey T, Wilson A, Williamson PM, O'Sullivan DJ, Rail D, et al. Reliability of the Columbia scale for assessing signs of parkinson's disease. Mov Disord. 1993;8(4):466-72.

7. Van Hilten JJ, Van Der Zwan AD, Zwinderman AH, Roos RAC. Rating impairment and disability in parkinson's disease: evaluation of the unified Parkinson's Disease Rating Scale. Mov Disord. 1994;9(1):84-8.

8. Kuopio A, Marttila RJ, Helenius H, Toivonen M, Rinne UK. The quality of life in parkinson's disease. Mov Disord. 2000;15(2):216-23.

9. Dural A, Atay M, Akbostanci C, Kucukdeveci A. Impairment, disability, and life satisfaction in parkinson's disease. Disabil Rehabil. 2003;25(7):318-23.

10. Deane KH, Jones D, Playford ED, Ben-Shlomo Y, Clarke CE. Physiotherapy for patients with Parkinson's disease: a comparison of techniques. Cochrane Database Syst Rev. 2001;(3):CD002817.

11. Greenberg DA, Aminoff MJ, Simon RP. Neurologia clínica. 5a ed. Porto Alegre: Artemed; 2005.

12. Camargos ACR, Cópio FCQ, Sousa TRR, Goulart F. O impacto da doença de parkinson na qualidade de vida: uma revisão de literatura. Rev Bras Fisioter. 2004;8(3):267-72.

13. Souza GFM, Moura TP, Cãmara NJD, Lima CF. Doença de parkinson, aspectos clínicos: visão da fisioterapia. Fisioter Mov. 2006;19(3):27-34.

14. Marinus J, Ramaker C, Van Hilten JJ, Stiggelbout AM. Health related quality of life in Parkinson's disease: a systematic review of disease specific instruments. J Neurol Neurosurg Psychiatry. 2002;72(2):241-8.

15. Hobart JC, Lamping DL, Thompson AJ. Evaluating neurological outcome measures: the bare essentials. J Neurol Neurosurg Psychiatry. 1996;60(1):127-30.

16. Rothstein JM. Measurement and clinical practice: theory and application. In: Rothstein JM. editor. Measurement in physical therapy. New York: Churchill Livingstone; 1985. p. 1-46.

17. Rodrigues GF, Barbosa CM, Silva CM, Teixeira SL, Cardoso F. O impacto de um programa de atividade física na qualidade de vida de pacientes com doença de parkinson. Rev Bras Fisioter. 2005;9(1):49-55.

18. Horta W. Escalas clínicas para avaliação de pacientes com doença de parkinson. In: Meneses Ms, Teive HAG. Doença de Parkinson: aspectos clínicos e cirúrgicos. Rio de Janeiro: Guanabara Koogan; 1996. p. 83-96.

19. Martignoni E, Franchignoni F, Pasetti C, Gerriero G, Picco D. Psychometric properties of the unified Parkinson's disease rating scale and of the short Parkinson's evaluation scale. Neurol Sci. 2003;24:190-1. 
20. Schrag A, Jahanshahi M, Quinn N. What contributes to quality of lifein patients with parkinson's disease? J Neurol Neurosurg Psychiatry. 2000;69(3):308-12.

21. Peto V, Jenkinson C, Fitzpatrick R. PDQ-39: a review of the development, validation and application of a parkinson's disease quality of life questionnaire and its associated measures. J Neurol. 1998;245(Suppl 1):S10-4.

22. Nieuwboer A, De Weerdt W, Dom R, Bogaerts K, Nuyens G. Development of an activity scale for individuals with advanced parkinson's disease: reliability and "on-off” variability. Phys Ther. 2000;80(11):108796.

23. Hunt S, McEwen J, Mckennas S. Measuring health status: a new tool for clinicans and epidemiologists. J R Coll Gen Pract. 1985;35(273):185-8.

24. Haase DCBV, Machado DC, Oliveira JGD. Atuação da fisioterapia no paciente com doença de parkinson. Fisioter Mov. 2008;21(1):79-85.

25. Sampaio RF, Mancini MC, Fonseca ST. Produção científica e atuação profissional: aspectos que limitam essa integração na fisioterapia e na terapia ocupacional. Rev Bras Fisioter. 2002;6(3):113-8.

26. Shenkman ML, Clark K, Xie T, Kuchibhatla M, Shinberg M, Ray L. Spinal movement and perfomance of standing reach task in participants with and without parkinson disease. Phys Ther. 2001;81(8):1400-11.

27. Jordan N, Harvey JS, Cooper JA. A component analysis of the generation and release of isometric force in parkinson's disease. J Neurol Neurosurg Psychiatry. 1992;55(7):572-6.

28. Goulart F, Santos CC, Teixeira-Salmela LF, Cardoso F. Análise do desempenho funcional em pacientes portadores de doença de parkinson. Acta Fisiatr. 2004;11(1):12-6.

29. Damiano AM, Snyder C, Strausser B, Willian MK. A review of health-related quality-of-life concepts and measures for Parkinson's disease. Qual Life Res. 1999;8(3):235-43.

30. Jenkinson C, Fitzpatrick R, Peto V, Greenhall R, Hyman N. The Parkinson's disease questionnaire (PDQ-39): development and validation of a parkinson's disease summary index score. Age Ageing. 1997;26(5):353-7.

31. De Boer A, Wijker W, Speelman JD, De Haes JCJM. Quality of life in patients with parkinson's disease: development of a questionnaire. J Neurol Neurosurg Psychiatry. 1996;61(1):70-4.

Recebido: 26/01/2009

Received: $01 / 26 / 2009$

Aprovado: 27/10/2009

Approved: 10/27/2009 